\title{
NP-Logic Systems and Model-Equivalence Reductions *
}

\author{
Yuping Shen \\ Institute of Logic and Cognition \\ Sun Yat-Sen University \\ 510275 Guangzhou, P. R. China \\ shyping@mail.sysu.edu.cn
}

\author{
Xishun Zhao \\ Institute of Logic and Cognition \\ Sun Yat-Sen University \\ 510275 Guangzhou, P. R. China \\ hsszxs@mail.sysu.edu.cn
}

In this paper we investigate the existence of model-equivalence reduction between NP-logic systems which are logic systems with model existence problem in NP. It is shown that among all NP-systems with model checking problem in NP, the existentially quantified propositional logic $(\exists \mathrm{PF})$ is maximal with respect to poly-time model-equivalent reduction. However, $\exists \mathrm{PF}$ seems not a maximal NPsystem in general because there exits a NP-system with model checking problem $D^{P}$-complete.

\section{Introduction}

For a complexity class $\mathscr{C}$, there are many logic systems for which the model existence problem (i.e. the satisfiability problem) lies in $\mathscr{C}$. We call such systems $\mathscr{C}$-systems. Take NP as an example, the following logic systems are all NP-systems:

- PF, the class of propositional formulas,

- CNF, the class of propositional formulas in conjunctive normal form,

- $k \mathrm{CNF}$, the class of CNF-formulas in which each clauses contains at most $k$ literals, where $k \geq 3$.

- LP, the class of normal logic programs with answer set semantics [2].

- $\exists \mathrm{PF}$, the class of quantified Boolean formulas with only existential quantifiers.

Among the above systems we have the following observations:

- All systems in $\{\mathrm{PF}, \mathrm{CNF}, \mathrm{LP}, \exists \mathrm{PF}\}$ have the same expressive power w.r.t. equivalence. More precisely, for any two systems $\mathscr{S}_{1}, \mathscr{S}_{2} \in\{\mathrm{PF}, \mathrm{CNF}, \mathrm{LP}, \exists \mathrm{PF}\}$, there is a transformation which translates every formula in $\mathscr{S}_{1}$ to a formula in $\mathscr{S}_{2}$ such that the two formulas are equivalent (i.e., they have the same models).

- From $(k+1) \mathrm{CNF}$ to $k \mathrm{CNF}$ there is no transformation which preserves the equivalence. Further, CNF has strictly stronger expressive power than $k \mathrm{CNF}$ (see e.g. [4]).

- From PF to CNF there is no poly-space transformation which preserves the equivalence (see e.g. [4]).

- Under the conjecture $\mathrm{P} \not \subset \mathrm{NC}^{1} /$ poly (see [7]), there is no poly-space transformation from LP to PF which preserves the equivalence [5].

From the above we can see that the expressive power of logic systems in the same complexity class are quite different. Since poly-space transformations preserving equivalence do not exist between some $\mathrm{NP}$-systems, it is quite natural to investigate the existence of (poly-time or poly-space) reductions which

${ }^{*}$ Research was partially supported by the NSFC projects under grant No. 60970040

X. Zheng and N. Zhong (Eds.)

Computability and Complexity in Analysis (CCA 2010)

EPTCS 24, 2010, pp. 130138 doi 10.4204/EPTCS.24.17 (c) Y. Shen \& X. Zhao

This work is licensed under the Creative Commons Attribution License. 
only preserve some relaxed equivalence. One of such reductions called model-equivalent reduction was introduced by Xishun Zhao and Kleine Büning in [11]. Informally speaking, a system $\mathscr{S}$ can be modelequivalently reduced to $\mathscr{S}^{\prime}$ if every formula $F$ in $\mathscr{S}$ can be transformed into a formula $F^{\prime}$ in $\mathscr{S}^{\prime}$ such that there is a poly-time computable one-to-one correspondence between the models of $F$ and $F^{\prime}$. With respect to poly-time model-equivalent reduction, systems PF, CNF, 3CNF, LP have the same expressive power (see [11] [6]). However, $\exists \mathrm{PF}$ still has strictly stronger expressive power than $\mathrm{PF}$ under the conjecture that $\mathrm{NP} \nsubseteq \mathrm{P} /$ poly which is widely believed true. That is, there seems no even poly-space model-equivalent reduction from $\exists \mathrm{PF}$ to PF. So, the authors of [11] asked whether $\exists \mathrm{PF}$ is a maximal NP-system w.r.t. poly-time model-equivalent reduction. This paper is concerned with this question. The remainder is organized as follows. In section 2, for the convenience of proof of our main result, we give a general but formal definition of logic systems. After listing some examples of logic systems, we reformulate the definition of model-equivalent reduction. In section 3, the main results are proved. More precisely, we prove the following: Any NP-system with model checking problem in NP can be poly-time model-equivalently to $\exists \mathrm{PF}$, The model checking problem is to decide whether a given formula is satisfied by a given interpretation. However, there do exist a NP-system for which the model checking problem is co-NP-complete or even harder. Then we show that there is a NP-system which is incomparable with $\exists \mathrm{PF}$, and that there is a NP-system with strictly stronger expressive power than $\exists \mathrm{PF}$ under a conjecture in complexity theory.

\section{Logic System and Model-equivalent Reduction}

Roughly speaking, a logic system consists of three parts, the language which is usually identified with the class of formulas constructed from the symbols in the language, the semantics which consists of all possible interpretations of symbols in the language, and the deductive relation. However, for our purpose we adopt the following formal definition.

Definition 1 A logic system is a tuple $(\Gamma, \Delta, T, S, R)$ satisfying the following conditions:

- $\Gamma, \Delta$ are non-empty finite sets of symbols, and $\Gamma \cap \Delta=\emptyset$,

- $T \subseteq \Gamma^{*}, S \subseteq \Delta^{*}$, both are poly-time decidable, and

- $R \subseteq \Gamma^{*} \times \Delta^{*}$ is a binary relation.

For a string $t \in T$, and a string $w \in S$, we say $w$ is a $R$-model of $t$ if $R(t, w)$ holds. The set of all $R$-models of $t$ is denoted as $\operatorname{Mod}_{R}(t)$

Intuitively, one may regard strings in $T$ as (encodings of) finite theories (e.g., a propositional formula, or a logic program, etc.), whereas strings in $S$ are intended to encode interpretations of atoms. Then the predicate $R(t, w)$ says the interpretation encoded by $w$ satisfies the theory encoded by $t$. That is, $R$ is a satisfactory relation. Please note that from the satisfactory relation $R$ we can define the following deductive relation: we say $t_{1}$ entails $t_{2}$ if $\operatorname{Mod}_{R}\left(t_{1}\right) \subseteq \operatorname{Mod}_{R}\left(t_{2}\right)$.

Example 1. Let $\mathscr{L}:=\{x, \mid, \neg, \wedge, \vee, \rightarrow, \exists, \forall),(\},, \Delta=\{0,1\}$. We intend to use $x|, x \|, x|||, \cdots$ to denote the propositional variables $x_{1}, x_{2}, x_{3}, \cdots$. A string $t$ of $\mathscr{L}^{*}$ is an encoding of a propositional formula over variables $x_{1}, \cdots, x_{n}$ if $t$ can be obtained from the formula by replacing each occurrence of each $x_{i}$ by $x|\cdots|$ ( $x$ is followed by $i$ many |'s). Similarly, logic programs, quantified Boolean formulas can be encoded in a natural way as strings in $\mathscr{L}^{*}$.

Further, $\{0,1\}$-sequences $w$ with length $n$ are intended to code truth assignments $v$ on variables $x_{1}, x_{2}, \cdots, x_{n}$, more precisely, $v\left(x_{i}\right)=1$ if and only if the $i$-th symbol in $w$ is 1 . Please note that a truth 
assignment is uniquely determined by a subset of atoms and vice visa. Thus we can also consider a $\{0,1\}$-sequence as an encoding of a subset of atoms.

(1) Let PF be the class of encodings of propositional formulas, TA: $=\{0,1\}^{*}$, and $\operatorname{Sat}(t, w)$ be the relation which says that $w$ is an encoding of a satisfying truth assignment of the formula coded by $t$. Then $(\mathscr{L},\{0,1\}$, PF, TA, Sat $)$ is in fact the propositional logic system.

(2) Let LP be the class of encodings of (propositional) logic programs, $\operatorname{ANS}(t, w)$ be the relation which says that $w$ is an encoding of an answer set (see [2]) of the normal logic program coded by $t$. Then $(\Gamma,\{0,1\}, \mathrm{LP}, \mathrm{TA}, \mathrm{ANS})$ is the answer set logic programming system.

(3) Let $\exists$ PF be the class of encodings of formulas $\Phi$ of the form $\exists x_{1} \cdots \exists x_{m} \varphi$ with $\varphi \in$ PF (here free variables are allowed). And let $\operatorname{FSat}(\Phi, w)$ be the relation which says that $w$ is an encoding of a truth assignment $v$ on free variables of $\Phi$, and after applying $v$ to $\Phi$ the resulting formula $\Phi[v]$ is true. Then $(\mathscr{L},\{0,1\}, \exists \mathrm{PF}, \mathrm{TA}$, Fsat) is a logic system.

For simplicity, from now on we write a logic system $(\Gamma, \Delta, T, S, R)$ just as $(T, S, R)$. For example we will write (PF, TA, Sat) instead of ( $\mathscr{L},\{0,1\}$, PF, TA, Sat).

Definition 2 ([11] $)$ Let $\left(T_{1}, S_{1}, R_{1}\right),\left(T_{2}, S_{2}, R_{2}\right)$ be two logic systems. We say $\left(T_{1}, S_{1}, R_{1}\right)$ can be polytime model-equivalently reduced to $\left(T_{2}, S_{2}, R_{2}\right)$, denoted as $\left(T_{1}, S_{1}, R_{1}\right) \preceq_{\text {ptime }}\left(T_{2}, S_{2}, R_{2}\right)$, if there are two polynomials $p(n)$ and $q(n)$, a function $f: T_{1} \longrightarrow T_{2}$, and a mapping $g: T_{1} \times S_{1} \longrightarrow S_{2}$ satisfying

- $f$ is computable in time $p(n)$, where $n$ is the size of input theory $t$,

- $g$ is computable in time $q(n)$, where $n$ is the size of input $(t, w)$, and

- for any fixed $t \in T_{1}$, the mapping $g_{t}$, defined by $g_{t}(v):=g(t, v)$, is a bijection from $\operatorname{Mod}_{R_{1}}(t)$ to $\operatorname{Mod}_{R_{2}}(f(t))$.

If in the above definition of $\preceq_{\text {ptime }}$ we replace " $f$ is computable in time $p(n)$ " by " $f$ is computable in space $p(n)$ ", then the reduction is called poly-space model-equivalent reduction, denoted as $\preceq_{\text {pspace }}$.

If the mapping $g$ in the definition of $\preceq$ ptime satisfies $g(t, v)=v$ for all $t \in T_{1}$ and $v \in S_{1}$, i.e., $t$ and $f(t)$

are equivalent, then the reduction is called poly-time equivalent reduction, denoted as $\preceq_{\text {ptime }}^{e q u}$. Likewise for poly-space equivalent reduction $\preceq_{\text {equace }}$.

Clearly, $\preceq_{\text {ptime }}$ and $\preceq_{\text {pspace }}$ are transitive. And a poly-time model-equivalent reduction is also a poly-space model-equivalent reduction.

\section{Lemma 1}

(1) $(P F, T A$, Sat $) \preceq_{\text {ptime }}(C N F, T A$, Sat $)$. [9]

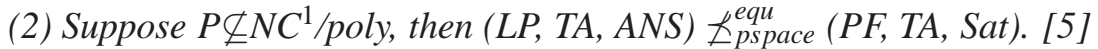

(3) Suppose NPÆP/poly, then ( $\exists P F, T A, F S a t) \npreceq$ pspace (PF, TA, Sat). [11]

\section{NP-Logic System}

For a logic system $(T, S, R)$, a theory $t \in T$ may have $R$-models with super-polynomial size in the size of $t$. However, in this paper we concentrate on systems such that any $R$-model of every theory has polynomial size. 
Definition 3 Suppose $(\Gamma, \Delta, T, S, R)$ is a logic system. If there is a polynomial $p$ such that $R(t, w)$ implies $|w|=p(|t|)$ for any $t \in T$ and any $w \in S$, then we call $(\Gamma, \Delta, T, S, R)$ a poly-size system.

Obviously, all systems in Example 1 are poly-size systems. From now on, whenever speaking of a logic system we mean it is a poly-size system.

Definition 4 A logic system $(T, S, R)$ is said to be a NP-logic system if the model-existence problem is in $N P$, i.e., the problem whether a given formula in $T$ has a R-model can be decided non-deterministically in polynomial time.

Obviously, for a logic system $(T, S, R)$, if the model-checking problem is in NP (i.e. $R$ is in NP) then the system is an NP-system. Therefore, all logic systems in Example 1 are NP-systems.

Theorem 1 (1) For any logic system $(\Gamma, \Delta, T, S, R)$, if the relation $R$ is decidable in polynomial time on a non-deterministic Turing machine, then $(T, S, R) \preceq_{\text {ptime }}(\exists P F, T A, F S a t)$

(2) For any logic system $(\Gamma, \Delta, T, S, R)$, if the relation $R$ is decidable in polynomial time on a deterministic Turing machine, then $(T, S, R) \preceq$ ptime (PF, TA, Sat).

Proof: (1) At first we have to construct a transformation from $T$ to PF. We shall adopt the construction in the proof of Cook-Levin theorem (see e.g. [10]) which states that the satisfiability problem for propositional formulas is NP-complete. Let $R^{\prime} \subseteq \Gamma^{*} \times \Delta^{*}$ be the relation defined by $R^{\prime}(t, w)$ if and only if $t \in T, w \in S$ and $R(t, w)$. Since $T, S$ are both decidable in polynomial time, $R^{\prime}$ is still decidable nondeterministically in polynomial time. Let $N=\left(\Gamma \cup \Delta, Q, \Gamma^{\prime}, \sigma, q_{0}, q_{\text {accept }}, q_{\text {reject }}\right)$ be a non-deterministic Turing machine that decides $R^{\prime}(t, w)$ in time $(|t|+|w|)^{k_{0}}$ for some constant $k_{0}$. Please note that we have assumed that $(T, S, R)$ is poly-size system (see Definition 3 ). Then there is a polynomial $p(n)$ such that $R(t, w)$ implies $|w|=p(|t|)$. Then on input $t, w$ with $|w|=p(|t|)$, the configurations of a branch of the computation can be represented as an $\left((|t|+p(|t|))^{k_{0}}+1\right) \times\left((|t|+p(|t|))^{k_{0}}+1\right)$ table. As shown in the following figure, the first row of the table is the starting configuration of $N$ on input $w, t$, and each row follows the previous one according to the transition function $\sigma$.

\begin{tabular}{|c|c|c|c|c|c|c|c|c|c|}
\hline$q_{0}$ & $t_{1}$ & $\cdots$ & $t_{n}$ & $w_{1}$ & $\cdots$ & $w_{m}$ & $\sqcup$ & $\cdots$ & $\sqcup$ \\
\hline & & & & & & & & & \\
\hline & & & & & & & & & \\
\hline & & & & & & & & & \\
& & & & & & & & & \\
& & & & & & & & & \\
& & & & & & & & & \\
& & & & & & & & & \\
\hline & & & & & & & & & \\
\hline
\end{tabular}

start configuration

second configuration

$(|t|+p(|t|))^{k_{0}}$ th configuration

For any $i, j$ with $1 \leq i, j \leq(|t|+p(|t|))^{k_{0}}+1$ and for each symbol $s \in \Gamma^{\prime} \cup Q$, we have a propositional variable $x_{i, j, s}$. If $x_{i, j, s}$ take the value 1 , it means that the entry (or cell) in row $i$ and column $j$ contains the symbol $s$.

In the proof of Cook-Levin theorem, for input $t, w$, four propositional formulas $\varphi_{\text {cell }}, \varphi_{\text {start }}, \varphi_{\text {move }}$, and $\varphi_{\text {accept }}$ are designed so that the Turing machine accepts $w, t$ if and only if $\varphi_{\text {cell }} \wedge \varphi_{\text {start }} \wedge \varphi_{\text {move }} \wedge \varphi_{\text {accept }}$ is satisfiable. 
$\varphi_{\text {start }}$ states that the first row of the table is the starting configuration of $N$ on input $t=t_{1} t_{2} \cdots t_{n}, w=$ $w_{1} w_{2} \cdots w_{p(n)}$. More precisely,

$$
\begin{aligned}
\varphi_{\text {start }}:= & x_{1,1, q_{0}} \wedge x_{1,2, t_{1}} \wedge \cdots \wedge x_{1, n+1, t_{n}} \wedge \\
& x_{1, n+2, w_{1}} \wedge \cdots \wedge x_{1, n+p(n)+1, w_{p(n)}} \wedge \\
& x_{1, n+p(n)+2, \sqcup} \wedge \cdots \wedge x_{1, n^{\prime}, \sqcup} .
\end{aligned}
$$

Here $n^{\prime}$ is $(n+p(n))^{k_{0}}+1$.

We need not to write explicitly other three formulas, instead we just explain their intuitive meaning. $\varphi_{\text {cell }}$ states that each cell contains exactly one symbol. The formula $\varphi_{\text {move }}$ guarantees that each row of the table corresponds to a configuration that can be obtained from the preceding row's configuration by applying a rule of $N$. Finally, $\varphi_{\text {accept }}$ states that an accepting configuration occurs during the computation.

Please note that the above construction of $\varphi_{\text {start }}$ depends on the input information $t, w$. However, our task is to construct a mapping which transforms each $t \in T$ to an existentially quantified formulas. That is, our construction should not depend on $w$. For that reason, we have to modify the formula $\varphi_{\text {start }}$. Please note the $w$ could be any string in $\Gamma^{*}$ with length $p(|t|)$. Hence, each cell in the first row and the $(n+1+j)$-th (with $1 \leq j \leq p(n)$ ) column could contain any symbol of $\Gamma \cup \Delta$. This can be described as the following formula:

$$
\alpha:=\left(\bigwedge_{n+2 \leq i \leq n+p(n)+1}\left(\bigvee_{s \in \Gamma \cup \Delta} x_{1, i, s}\right)\right)
$$

Now we define $\varphi_{\text {start }}^{\prime}$ as

$$
\varphi_{\text {start }}^{\prime}:=x_{1,1, q_{0}} \wedge x_{1,2, t_{1}} \wedge \cdots \wedge x_{1, n+1, t_{n}} \wedge \alpha \wedge x_{1, n+p(n)+2, \sqcup} \wedge \cdots \wedge x_{1, n^{\prime}, \sqcup}
$$

We write $G(t)$ to denote the formula $\varphi_{\text {cell }} \wedge \varphi_{\text {start }}^{\prime} \wedge \varphi_{\text {move }} \wedge \varphi_{\text {accept }}$. Clearly, $t$ has a $R$-model if and only if $G(t)$ is satisfiable. Please note that for a string $w$, the truth values of $x_{1, j, s}$ (with $1 \leq j \leq n^{\prime}$ ) can be uniquely determined by $t, w$ and formulas $\varphi_{\text {cell }}$ and $\varphi_{\text {start }}^{\prime}$, however, the truth value of each of the other variables is not uniquely determined due to the non-determinism of $N$. Thus, the models of $t$ do not necessarily one-to-one correspond to the truth assignments of $G(t)$. Therefore, we add existential quantifiers $\exists x_{i, j, s}$ in front of $G(t)$ for all $i=2, \cdots, n^{\prime}, j=1, \cdots, n^{\prime}$ and $s \in \Gamma \cup Q$, the resulting formula is denoted as $F(t)$. Now, it is easy to see that there is a polynomial-time computable one-to-one correspondence between $R$-models of $t$ and models of $F(t)$.

(2) Suppose $R$ is decidable deterministically in polynomial time. Then, in the above construction, we can assume that $N$ is a deterministic Turing machine deciding $R^{\prime}$. Since the computation of $N$ is uniquely determined whenever the input is fixed, we can see that $G$ is in fact a model-equivalence reduction from $(\Gamma, \Delta, T, S, R)$ to $(\mathscr{L},\{0,1\}, \mathrm{PF}, \mathrm{TA}, \mathrm{Sat})$.

Theorem 1 says that $\exists \mathrm{PF}$ is the maximal system amongst NP-systems for which the model checking problem is in NP. However, it is unlikely a maximal NP-system in general because there are NP-systems for which the model checking problem is co-NP-complete or even harder (under the assumption that the polynomial hierarchy does not collapse).

\section{Example 2.}

(1) Let MinSat $(t, w)$ be the relation which says that $w$ is an encoding of a minimal model of the propositional formula with code $t$. Here by a minimal model of a propositional formula $\varphi$, we mean a model $M$ of $\varphi$ such that any proper subset of $M$ is not a model of $\varphi$. Since a propositional formula 
$\varphi$ has a model if and only if $\varphi$ has a minimal model, it follows that $(\mathscr{L},\{0,1\}$, PF, TA, MinSat) is a NP-system.

(2) $(\mathscr{L},\{0,1\}, \exists$ PF, FMinSat) is a NP-system. Here FMinsat $(t, w)$ says $w$ is an encoding of a minimal model of the existentially quantified formula coded by $t$.

Proposition 1 (1) The model checking problem for (PF, TA, MiniSat) is co-NP-complete [1].

(2) The model checking problem for $\left(\exists P F, T A\right.$, FMiniSat) is $D^{P}$-complete. Where $D^{P}$ is the class of decision problems which can be described as the intersection of one NP problem and one co-NP problem [8].

Proof: (1) Please see page 48-49 in [1].

(2) At first we show the membership. Consider an arbitrary $\exists P F$ formula $\Phi$. Suppose the set of free (i.e. not quantified) variables in $\Phi$ is $Z=:\left\{z_{1}, \cdots, z_{n}\right\}$, and the set of bounded variables in $\Phi$ is $X:=$ $\left\{x_{1}, \cdots, x_{m}\right\}$. For simplicity we write $\Phi$ as $\exists X \varphi(X, Z)$. It is not hard to see that a subset $M \subseteq Z$ of is a minimal model of $\Phi$ if and only if $M$ is a model of the following formula.

$$
\exists X \varphi(Z, X) \wedge \forall Z^{\prime}\left(\neg\left(Z^{\prime} \rightarrow Z\right) \vee \forall X \neg \varphi\left(Z^{\prime}, X\right) \vee\left(Z^{\prime}=Z\right)\right) .
$$

Where $Z^{\prime}=\left\{z^{\prime} \mid z \in Z\right\}$ is a set of new variables; $Z^{\prime} \rightarrow Z$ is abbreviated for the formula $\bigwedge_{z \in Z}\left(z^{\prime} \rightarrow z\right)$; $Z^{\prime}=Z$ denotes the formula $\left(Z^{\prime} \rightarrow Z\right) \wedge\left(Z \rightarrow Z^{\prime}\right)$; and $\varphi\left(Z^{\prime}, X\right)$ is the formula obtained from $\varphi(Z, X)$ by replacing each occurrence of $z$ by $z^{\prime}$. It follows obviously that minimal model checking problem for $\exists \mathrm{PF}$ is in $D^{P}$.

Next we show the hardness. The canonical $D^{P}$-complete problem is the SAT-UNSAT problem (see [8]) of determining for a pair $(\varphi, \psi)$ of propositional formulas, whether $\varphi$ is satisfiable and $\psi$ is unsatisfiable. Let $X, Y$ be the sets of variables in $\varphi$ and $\psi$, respectively. We assume w.o.l.g. that $X \cap Y=\emptyset$. Let $z$ be a new variable. Consider the following formula

$$
F=\exists X \exists Y(\varphi \wedge(z \vee \psi)) .
$$

It is not hard to see that $(\varphi, \psi) \in$ SAT-UNSAT if and only if $\{z\}$ is a minimal model of $F$. The proof completes.

Theorem 2 Suppose co-NP $\neq N P$. Then (PF, TA, MinSat) and ( $\exists P F, T A, F S a t)$ are pairwise incomparable with respect to poly-time model-equivalence reduction.

Proof: The theorem follows from the following fact. The the minimal model checking problem for propositional formulas is co-NP-complete (see Proposition 1), whereas the model checking problem for existentially quantified propositional formulas is NP-complete [11]. Suppose for example (PF, TA, MinSat) $\preceq_{\text {ptime }}(\exists \mathrm{PF}, \mathrm{TA}, \mathrm{FSat})$. Then for a truth assignment $M$ and a propositional formula $\varphi$, to check that $M$ is a minimal model of $\varphi$, we first transform $\varphi$ in poly-time into a $\exists$ PF-formula $\Phi$, and compute $M^{\prime}$ from $M$ by using the poly-time computable one-to-one correspondence, then check that $M^{\prime}$ is a model of $\Phi$, which is a NP problem. It follows that $\mathrm{NP}=$ co-NP, contradicts the assumption of the theorem.

$\mathrm{NP} \nsubseteq \mathrm{P} /$ poly is an important conjecture in computational complexity theory (see e.g. [7]). In fact we even do not know whether NP $\nsubseteq$ co-NP/poly is true or false. However, the following theorem shows that if (PF, TA, MinSat) and ( $\exists \mathrm{PF}, \mathrm{TA}, \mathrm{FSat})$ are comparable with respect to poly-space model-equivalent reduction then $\mathrm{NP} \subseteq$ co-NP/poly.

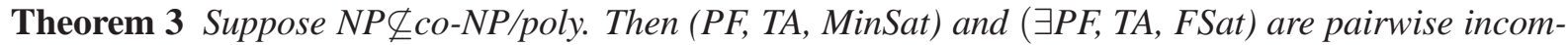
parable with respect to poly-space model-equivalent reduction. 
Proof: We first show ( $\exists$ PF, TA, FSat $\npreceq_{\text {pspace }}(\mathrm{PF}, \mathrm{TA}, \mathrm{MinSat})$. Let $\Gamma_{n}$ be the set of all 3CNF formulas $\varphi$ such that variables $|\varphi|=n$ and $\operatorname{var}(\varphi) \subseteq\left\{x_{1}, \cdots, x_{n}\right\}$, where $\operatorname{var}(\varphi)$ is the set of all variables occurring in $\varphi$. Define $\Gamma:=\bigcup_{n>0} \Gamma_{n}$. Clearly, the satisfiability problem for $\Gamma$ is NP-complete.

Let $\pi(n)$ be the set of 3-clauses over $x_{1}, \cdots, x_{n}$. For each 3-clause $c \in \pi(n)$ introduce a new variable $z_{c}$. Define

$$
\Psi_{n}:=\exists x_{1} \cdots \exists x_{n}\left(\bigwedge_{c \in \pi(n)}\left(c \vee \neg z_{c}\right)\right)
$$

Let $\varphi$ be a 3CNF formula with $|\varphi|=n$. W.1.o.g. we can assume $\varphi \in \Gamma_{n}$. Suppose $\varphi=c_{1} \wedge \cdots \wedge c_{k}$. Define $M_{\varphi}=\left\{z_{c_{1}}, \cdots, z_{c_{k}}\right\}$, that is, we set each $z_{c_{i}}$ to 1 , and all other $z_{c}$ to 0 . Clearly,

- $M_{\varphi}$ can be computed in polynomial time, and

- $\varphi$ is satisfiable if and only if $M_{\varphi}$ is a model of $\Psi_{n}$.

Suppose $(\exists \mathrm{PF}, \mathrm{TA}, \mathrm{FSat}) \preceq_{\text {pspace }}\left(\mathrm{PF}, \mathrm{TA}\right.$, MinSat). Then there is a sequence $\psi_{1}, \psi_{2}, \cdots, \psi_{n}, \cdots$ of propositional formulas such that

- the size of each $\psi_{n}$ is bounded by a polynomial, and

- for each $n$, there is a polynomial-time computable one-to-one correspondence between the models of $\Psi_{n}$ and $\psi_{n}$.

Then we define an advice-taking Turing machine 1 in the following way. The advice oracle is $\psi_{n}$. Given an instance $\varphi$ of $\Gamma$ with $|\varphi|=n$, the machine loads $\psi_{n}$, then computes $M_{\varphi}$ in polynomial time in $n$, then computes $M_{\varphi}^{\prime}$ according to the one-to-one correspondence, finally checks whether $M_{\varphi}^{\prime}$ is a minimal model of $\psi_{n}$. Please note that the minimal model checking problem for propositional formulas is in co-NP. Since the satisfiability problem for $\Gamma$ is NP-complete, it follows that NP $\subseteq$ co-NP/poly.

(2) Next we show (PF, TA, MinSat) $\npreceq_{\text {pspace }}(\exists \mathrm{PF}, \mathrm{TA}, \mathrm{FSat})$. Suppose $\Gamma_{n}, \Gamma, \pi(n), z_{c}$ are defined as before. Now for each $c \in \pi(n)$ we introduce another new variable $z_{c}^{\prime}$ for each $c \in \pi(n)$, and a new variable $y$ in addition. Define

$$
\psi_{n}:=\left(\left(\neg y \wedge \bigwedge_{c \in \pi(n)}\left(c \vee \neg z_{c}\right)\right) \vee\left(y \wedge x_{1} \wedge \cdots \wedge x_{n}\right)\right) \wedge\left(\bigwedge_{c \in \pi(n)}\left(z_{c} \leftrightarrow \neg z_{c}^{\prime}\right)\right) .
$$

Let $\varphi$ be a 3CNF formula with $|\varphi|=n$. W.1.o.g. we can assume $\varphi \in \Gamma_{n}$. Suppose $\varphi=c_{1} \wedge \cdots \wedge c_{k}$. Define

$$
M_{\varphi}=\left\{y, x_{1}, \cdots, x_{n}\right\} \cup\left\{z_{c_{1}}, \cdots, z_{c_{k}}\right\} \cup\left\{z_{c}^{\prime} \mid c \notin \varphi\right\}
$$

It is not hard to see that

- $M_{\varphi}$ can be computed in polynomial time, and

- $\varphi$ is unsatisfiable if and only if $M_{\varphi}$ is a minimal model of $\Psi_{n}$.

Suppose (PF, TA, MinSat) $\preceq_{\text {pspace }}(\exists \mathrm{PF}, \mathrm{TA}, \mathrm{FSat})$. Then there is a sequence $\Psi_{1}, \Psi_{2}, \cdots, \Psi_{n}, \cdots$ of $\exists \mathrm{PF}$ formulas such that

- the size of each $\Psi_{n}$ is bounded by a polynomial, and

- for each $n$, there is a polynomial-time computable one-to-one correspondence between the models of $\psi_{n}$ and $\Psi_{n}$.

\footnotetext{
${ }^{1}$ For a precise definition of advise-taking Turing machine please see [7].
} 
Then we define an advice-taking Turing machine in the following way. The advice oracle is $\Psi_{n}$. Given an instance $\varphi$ of $\Gamma$ with $|\varphi|=n$, the machine loads $\Psi_{n}$, then computes $M_{\varphi}$ in polynomial time in $n$, then computes $M_{\varphi}^{\prime}$ from $M_{\varphi}$ according to the one-to-one correspondence, finally checks whether $M_{\varphi}^{\prime}$ is a model of $\Psi_{n}$. Please note that the model checking problem for $\exists \mathrm{PF}$ formulas is in NP. Since the unsatisfiability problem for $\Gamma$ is co-NP-complete, it follows that $\mathrm{NP} \subseteq$ co-NP/poly.

Lemma 2 (1) (PF, TA, MinSat) $\preceq_{\text {ptime }}(\exists P F, T A$, FMinSat $)$.

(2) $(\exists P F, T A, F S a t) \preceq$ ptime $(\exists P F, T A, F M i n S a t)$.

Proof: (1) Directly follows from the fact that (PF, TA, MinSat) is a sub-system of ( $\exists$ PF, TA, FMinSat). (2) Consider any formula $\Phi=\exists y_{1} \cdots \exists y_{m} \varphi$ with free variables $x_{1}, \cdots, x_{n}$. Now we introduce for each $x_{i}$ $(i=1, \cdots, n)$ a new variable $x_{i}^{\prime}$ which is intended to stand for $\neg x_{i}$. Define $\varphi^{\prime}:=\varphi \wedge \wedge\left(\left(x_{i} \vee x_{i}^{\prime}\right) \wedge\left(\neg x_{i} \vee \neg x_{i}^{\prime}\right)\right)$ and let $\Phi^{\prime}:=\exists y_{1} \cdots \exists y_{n} \varphi^{\prime}$. Clearly a subset $M \subseteq\left\{x_{1}, \cdots, x_{n}\right\}$ is a model of $\Phi$ if and only if $M \cup\left\{x_{i}^{\prime} \mid x_{i} \notin\right.$ $M\}$ is a minimal model of $\Phi^{\prime}$.

Corollary 1 Suppose co-NP $\neq N P$. Then

(1) ( $\exists P F, T A, F M i n i S a t) \npreceq_{\text {ptime }}(P F, T A$, MinSat).

(2) $(\exists P F, T A, F M i n S a t) \npreceq$ ptime $(\exists P F, T A, F S a t)$.

Proof: If ( $\exists$ PF, TA, FMiniSat) $\preceq_{\text {ptime }}$ (PF, TA, MinSat) or ( $\exists$ PF, TA, FMinSat) $\preceq_{\text {ptime }}$ ( $\exists$ PF, TA, FSat), then we have by Lemma 2 that (PF, TA, MinSat) and ( $\exists$ PF, TA, FSat) are comparable w.r.t. poly-time model-equivalent reduction. This contradicts Theorem 2.

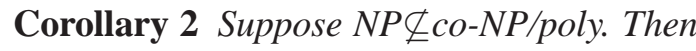

(1) $(\exists P F, T A, F M i n i S a t) \npreceq_{\text {pspace }}(P F, T A$, MinSat $)$.

(2)( $\exists P F, T A, F M i n S a t) \npreceq_{\text {pspace }}(\exists P F, T A, F S a t)$.

Proof: If ( $\exists$ PF, TA, FMiniSat) $\preceq_{\text {pspace }}$ (PF, TA, MinSat) or ( $\exists$ PF, TA, FMinSat) $\preceq_{\text {pspace }}(\exists \mathrm{PF}, \mathrm{TA}, \mathrm{FSat})$, then we have by Lemma 2 that (PF, TA, MinSat) and ( $\exists$ PF, TA, FSat) are comparable w.r.t. poly-space model-equivalent reduction. This contradicts Theorem 3.

\section{Conclusion and Future work}

We have proved that w.r.t. poly-time model-equivalent reduction ( $\exists \mathrm{PF}$, TA, FSat) has the strongest expressive power among NP systems with model checking problem in NP, whereas (PF, TA, Sat) is strongest among NP systems with model checking problem in P. However, ( $\exists$ PF, TA, FSat) is unlikely the strongest NP system, because it have been shown that ( $\exists \mathrm{PF}$, TA, FSat) $\preceq_{\text {ptime }}(\exists \mathrm{PF}$, TA, MinSat) but the converse in not true under the assumption NP $\nsubseteq$ co-NP. We conjecture that there is no strongest NP-system under some conjecture in computational complexity.

\section{References}

[1] M. Cadoli: Tractability Reasoning in Artificial Intelligence, Springer, Berlin, 1994.

[2] M. Gelfond, V. Lifschitz: The Stable Model Semantics for Logic Programming. In Proceedings of the 5th International Conference on Logic Programming, 1070-1080, The MIT Press, 1988. 
[3] R. Greenlaw, H.J. Hoover, and W.L. Ruzzo: Limits to Parallel Computation: P-Completeness theory. Oxford University Press, 1995.

[4] H. Kleine Büning and T. Lettmann: Propositional Logic: Deduction and Algorithms. Cambridge University Press, 1999.

[5] V. Lifschitz, A. Razborov: Why Are There So Many Loop Formulas, ACM Transactions on Computational Logic, 7: 261-268, 2006.

[6] Fangzhen Lin and Jicheng Zhao: On Tight Logic Programs and Yet Another Translation from Normal Logic Programs to Propositional Logic. In Proc. IJCAI-03, 853-858.

[7] C.H. Papadimitriou: Computational Complexity. Addison-Wesley, New York, 1994.

[8] C.H. Papadimitriou, D. Wolfe: The Complexity of Facets Resolved. Journal of Computer and System Sciences, 37: 2-12, 1988.

[9] D. Plaisted, S. Greenbaum: A Structure Preserving Clause Form Transformation, Journal of Symbolic Computation, 2(3): 293-304, 1986.

[10] M. Sipser: Introduction to the Theory of Computation, seond edition, China Machine Press, 2006.

[11] Xishun Zhao, H. Kleine Büning: Model-equivalent Reductions. In Lecture Notes in Computer Science 3569, 355-370, Springer, 2005.

[12] Xishun Zhao, Yuping Shen: Comparison of Semantics of Disjunctive Logic Programs Based on Modelequivalent Reduction. Journal of Computer Science and Technology, 22(4): 562-568, 2007. 